\title{
Clinical Profile, Dosing, and Quality-of-Life Outcomes in Primary Immune Deficiency Patients Treated at Home with Immunoglobulin G: Data from the IDEaL Patient Registry
}

\author{
Sean Kearns, PhD; Loretta Kristofek, BSN; William Bolgar, PharmD; \\ Luqman Seidu, MD; and Samantha Kile, BS
}

\begin{abstract}
BACKGROUND: Patients with primary immune deficiency (PID) often require immunoglobulin $\mathrm{G}$ (IgG, commonly referred to as Ig) replacement therapy to prevent infections and associated comorbidities. Ig therapy can be given either through intravenous or subcutaneous routes, and both can be done in the home setting. There is limited information available on the real-world diagnosis, management, and outcomes of this patient population, given the variable disease presentation and treatment options. The Immunoglobulin Diagnosis, Evaluation, and key Learnings (IDEaL) Patient Registry is designed to capture nursing, pharmacy, and patient-reported data for patients receiving $\mathrm{Ig}$ at home.
\end{abstract}

OBJECTIVES: To (a) present a real-world population of patients with PID who have received $\mathrm{Ig}$ at home and (b) examine how differences in administration, dosing, and insurance affect health and quality-of-life outcomes in these patients.

METHODS: As of July 2015, 383 patients receiving Ig therapy from Coram/ CVS specialty infusion services, across multiple disease states, signed consent forms and enrolled in the IDEaL Patient Registry. Patients' referral paperwork, including lab values, and standard of care nursing and pharmacy follow-up forms were collected. Patients were mailed quality-of-life surveys at the time of enrollment and every 6 months after their enrollment.

RESULTS: The most common diagnosis (78\%) in these PID patients was common variable immunodeficiency (CVID). For Ig-naive adult patients, the average age at the start of treatment was 59 years. For pediatric patients, average age at start of treatment was 9 years. A majority of these PID patients $(80 \%)$ received subcutaneous $\mathrm{Ig}(\mathrm{SCIg})$ at home, and $20 \%$ received intravenous Ig (IVIg). The average SClg dose was 10 grams per week, or $130 \mathrm{mg}$ per $\mathrm{kg}$, and the average IVlg dose was 36 grams every 4 weeks, or $472 \mathrm{mg}$ per $\mathrm{kg}$. In the IVIg patient population, $34 \%$ had a dose or frequency change while on treatment, while $30 \%$ of the SClg patients had a dose or frequency change. Patient-reported health and quality-of-life scores were generally positive. Route of administration did not affect patient perception of cost $(P=0.171)$, but whether the patient had private or governmentbacked health care did affect perception of cost $(P=0.036)$.

CONCLUSIONS: For a disease state with an extremely variable presentation, data from the IDEaL Patient Registry provides further insights into the realworld clinical and diagnostic characteristics of this population, as well as dosing and treatment outcomes of home administration of Ig therapy. The majority of patients received SCIg infusions. SCIg dosing was on the lower end of the recommended $\mathrm{mg}$ per $\mathrm{kg}$ dose range, while IVIg patients were more in the middle of the recommended dose range. Patient outcomes on treatment were correlated with baseline status, suggesting that earlier detection and treatment of primary immune deficiencies may be critical in achieving beneficial outcomes on Ig therapy.

J Manag Care Spec Pharm. 2017;23(4):400-06

Copyright $\odot 2017$, Academy of Managed Care Pharmacy. All rights reserved.

\section{What is already known about this subject}

Clinical trials for immunoglobulin (Ig) therapy in primary immune deficiency (PID) have established dose ranges where effective reductions in infections can be achieved.

Other studies have also shown that higher doses lead to higher Ig trough levels and that higher trough levels are correlated with lower rates of infections.

Previous reports have shown home infusion of Ig to be safe, with low levels of adverse events for intravenous Ig (IVIg) and subcutaneous Ig (SCIg).

\section{What this study adds}

This study provides real-world data on age at start of treatment, dosing, dose optimization, and compliance in patients being treated with IVIg and SCIg.

Patient-reported health status and health improvement while on treatment is reported, and these results suggest how initial health perception affects change in health status.

Patient perception of treatment cost is broken down by route of administration as well as payer type.

A lthough multiple clinical facets of primary immune deficiency (PID) are described in the medical literature, the high variability in demographics and clinical presentation in this population, and how it affects outcomes, presents challenges in treatment analysis. Purified immunoglobulin G (IgG, commonly referred to as Ig) is approved by the U.S. Food and Drug Administration for treatment of PID, and this diagnosis represents a substantial percentage of patients for whom Ig therapy is prescribed. ${ }^{1,2}$ The therapy goal is a demonstrable reduction in the incidence of infection, improved quality of life, and slowing or stopping the progression of associated organ damage. Reported incidence rates for PID vary from 1 in 1,200 in a nationwide telephone survey to 4.6 per 100,000 person-years in a historical cohort study of a single county in Minnesota over 30 years. ${ }^{3,4}$ Numerous reports have shown that a delay often occurs from initial presentation of symptoms to start of treatment with Ig, averaging from 4 to 6 years. ${ }^{4-6}$ Delays in diagnosing and 
treating the infections caused by PIDs substantially increase the risk for end-organ damage, impair quality of life, and increase mortality. ${ }^{5}$ This delay can create a significant financial burden on the patient and the health care system. ${ }^{6}$

The Immunoglobulin Diagnosis, Evaluation, and key Learnings (IDEaL) Patient Registry collects longitudinal information on a real-world population of patients receiving Ig replacement therapy from Coram/CVS specialty infusion services in alternate care settings across the United States. The IDEaL Registry differs from other immune deficiency registries in that it focuses on patients of multiple diagnoses receiving a variety of Ig brands via intravenous (IVIg) and subcutaneous (SCIg) administration routes, in alternate care settings (primarily at home). Home infusion is an increasingly used option for Ig therapy, in large part because of the rise of SCIg administration and the availability of trained home health care nurses. In addition to collecting information on outcomes among a heterogeneous population of PID patients, we also assessed how the Ig route of administration affects patient compliance and outcomes. The goal of the IDEaL Patient Registry is to examine data from real-world patients on Ig therapy, including information related to diagnosis, dosing, and therapy tolerance, and to highlight the broad range of patients on Ig treatment for PID.

\section{Methods}

The IDEaL Registry is approved by Schulman Associates Institutional Review Board. The study has 156 participating physicians across the country. Patients requiring Ig therapy who were referred to Coram/CVS specialty infusion services by the participating physicians were eligible to participate. Prospective subjects signed a written informed consent document either in person at the physician's office or via mail or phone by registry staff. Once enrolled, patients' treatment information from July 2010 onward was made available for entry into the IDEaL Registry database. Patient information included initial referral documentation and physician visit notes, nurse visit records for infusions, and pharmacist followup progress reports. Patients were mailed a Life Quality Index Questionnaire (LQIQ) and an SF-36 survey at the time of enrollment and every subsequent 6 months as long as they continued on Ig therapy. As of July 2015, 383 patients were enrolled in the study with longitudinal data collection going back approximately 4.5 years to the first subject enrolled after the study inception date of July 1, 2010.

\section{Results}

\section{Distribution of PID Diagnoses}

A total of 383 patients signed consent forms and enrolled in the registry as of July 2015. Of this patient population, $95 \%$ were diagnosed with PID $(n=367 / 383)$. The majority $(78 \%)$ of these PID patients were diagnosed with common variable immunodeficiency (CVID; International Classification of Diseases, Ninth

\section{TABLE 1 Immunoglobulin Diagnosis, Evaluation, and key Learnings (IDEaL) Patient Registry Demographics}

\begin{tabular}{|c|c|}
\hline Under 18 years & 9 years \\
\hline 18 years and over & 59 years \\
\hline \multicolumn{2}{|l|}{ Age distribution - allergy/immunology } \\
\hline Under 18 years & $33 / 367$ \\
\hline 18 years and over & $334 / 367 \quad(91 \%)$ \\
\hline 65 years and over & $128 / 367 \quad(35 \%)$ \\
\hline \multicolumn{2}{|l|}{ Gender distribution - allergy/immunology } \\
\hline Male & $89 / 367 \quad(24 \%)$ \\
\hline Female & $278 / 367 \quad(76 \%)$ \\
\hline \multicolumn{2}{|l|}{ Average dosing for SCIg and IVIg } \\
\hline SCIg average dose & $\begin{array}{l}520 \mathrm{mg} / \mathrm{kg} / \mathrm{month} \\
(130 \mathrm{mg} / \mathrm{kg} / \text { week })\end{array}$ \\
\hline IVIg average dose & $472 \mathrm{mg} / \mathrm{kg} / \mathrm{month}$ \\
\hline \multicolumn{2}{|l|}{ PID diagnosis ${ }^{\mathrm{a}}$ distribution } \\
\hline 279.0-Deficiency humoral immunity & $3 / 367(0.8 \%)$ \\
\hline 279.00-Hypogammaglobulinemia, unspecified & $41 / 367 \quad(12 \%)$ \\
\hline 279.03-Other selective immunoglobulin deficiency & $16 / 367 \quad(4 \%)$ \\
\hline 279.04-Congenital hypogammaglobulinemia & $4 / 367$ \\
\hline 279.05-Immunodeficiency w/hyper IgM & $1 / 367 \quad(0.2 \%)$ \\
\hline 279.06-Common variable immunodeficiency & $284 / 367 \quad(78 \%)$ \\
\hline 279.09-Other deficiency humoral immunity & $1 / 367(0.2 \%)$ \\
\hline 279.2-Combined immunity deficiency & $5 / 367(0.8 \%)$ \\
\hline 279.3-Unspecified immunity deficiency & $11 / 367$ \\
\hline
\end{tabular}

${ }^{a}$ Codes are from the International Classification of Diseases, Ninth Revision, Clinical Modification

Ig = immunoglobulin; IgM = immunoglobulin $M ; I V I g=$ intravenous immunoglobulin; PID = primary immune deficiency; $S C I g=$ subcutaneous immunoglobulin.

Revision, Clinical Modification [ICD-9-CM] code 279.06), with $12 \%$ diagnosed with unspecified hypogammaglobulinemia (ICD-9-CM code 279.00), and the remainder with other PID diagnoses (Table 1).

\section{Gender and Age Breakdown}

The PID population enrolled in the registry was predominately female $(76 \%, \mathrm{n}=278)$ and comprised the majority in all the age categories except for pediatric patients, where there were slightly more males enrolled. The pediatric population accounted for $9 \%$ of the total population ( $n=33$ ), while $56 \%$ of adults were aged under 65 years $(n=206)$, and $38 \%$ were aged 65 years and over $(n=128)$. We examined the average age at the start of treatment for those patients who were Ig-naive at the time of their referral. For patients aged under 18 years, the average age was 9 years. For patients aged 18 years and over, the average age was 59 years (Table 1 ).

\section{Route and Dosing}

For patients with at least 4 documented pharmacy doses and follow-ups, $80 \%(n=218)$ received SCIg with self-administration, 


\section{FIGURE 1 Dose and Frequency Changes in PID} Patients Receiving Ig Therapy

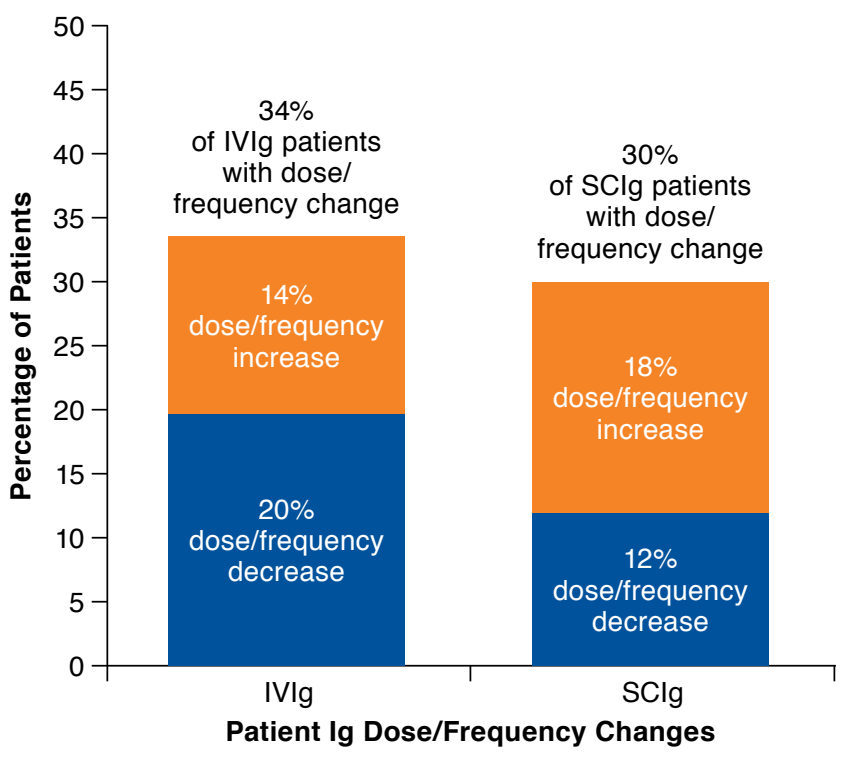

Ig = immunoglobulin; IVIg = intravenous immunoglobulin; PID = primary immune deficiency; SCIg=subcutaneous immunoglobulin.

while 20\% ( $n=56)$ received IVIg at home from a skilled nurse. In the SCIg population, the average weekly dose was approximately 10 grams (range $=2-20$ grams per week) equating to $130 \mathrm{mg}$ per $\mathrm{kg}(\mathrm{mg} / \mathrm{kg})$. Fourteen of these patients were on an every-other-week infusion schedule, with an average infusion dose of 16 grams (range $=4-24$ grams). For the patients receiving IVIg, the average monthly dose was 36 grams, which equated to $472 \mathrm{mg} / \mathrm{kg}$ (Table 1). Thirteen of these patients were on an every 2- to 3-week schedule, with an average infusion dose of 33 grams (range $=13-76$ grams).

There were 32 patients who switched infusion routes during their time on treatment. Of these, 22 patients switched from IVIg to SCIg, and 10 patients switched from SCIg to IVIg. For those patients going from IVIg to SCIg, the average dose conversion was 1:1.3 (range=1:0.8-2.0). For the patients on SCIg moving to IVIg, the average dose conversion was 1:1.05 (range $=1: 0.63-1.85$ ).

\section{Dose Optimization}

Dose and infusion frequency changes were also tracked. Figure 1 shows that $30 \%$ of the SCIg patients $(n=65)$ had a dose or frequency change that changed the weekly or monthly total dose. Of those SCIg patients with a dose change, $60 \%$ had dose increases $(n=39)$. For the IVIg patients, $34 \%$ had a dose or frequency change $(n=19)$, and $42 \%$ of these were dose increases $(n=8)$.

\section{Drug Compliance}

Patients were asked if they had any missed, delayed, or incomplete doses of their Ig. In the SCIg population, $6 \%$ of the doses were marked as missed $(n=236 / 3,758) ; 4 \%(n=140)$ were marked as delayed; and $0.4 \%(n=15)$ were marked as incomplete. In the IVIg population, $1 \%$ were marked as missed $(\mathrm{n}=13 / 1,108) ; 4 \%(\mathrm{n}=49)$ were marked as delayed; and $1 \%$ $(\mathrm{N}=7)$ were marked as incomplete.

\section{Life Quality Index Questionnaire}

The LQIQ consisted of 15 questions ranked from 1-7 (negative to positive) that asked patients to rate their treatment and infusion experience. We examined responses at baseline, 6 months, and 12 months for questions related to patient perception of the convenience and the cost of infusions.

\section{Perception of Infusion Convenience}

One of the primary advantages for patients receiving their infusions in their homes is the convenience of not having to travel to an infusion suite and being able to receive an infusion in comfort. In general, our patient population had a very favorable perception of the convenience of their infusions, with over $86 \%$ ( $n=158 / 182$ ) of the population having a positive perception of the convenience of in-home infusions at baseline. That positive perception was maintained through the 12-month time point, with $92 \%$ of reports $(n=108 / 118)$ showing a positive perception. We did not see any significant difference in the perception of convenience between SCIg and IVIg across all time points $(P>0.1)$, although we noted that between time points, IVIg did show a decrease in positive perception of convenience, and SCIg did see an increase in positive perception (Figure 2).

\section{Cost Perception}

Ig therapy is an expensive treatment, and patient out-of-pocket costs can have a tremendous effect on compliance and ability to continue on this long-term therapy. On average, the scores for patient perception of cost were the lowest of all of the individual LQIQ components across all time points, with $50 \%$ of patients reporting a positive perception of the cost ("not too expensive"), while 32\% had a negative perception ("seems to be too expensive") and $18 \%$ had a neutral perception.

When we compared therapy administration routes across all time points, we noted no significant difference in the cost perception between SCIg and IVIg, with 54\% of IVIg patients having a positive perception, and $48 \%$ of SCIg patients having a positive perception $(P=0.6)$. Additionally, we noted no significant difference between cost perception in terms of primary payer, either private insurance ( $47 \%$ positive) or a governmentsponsored plan such as Medicare or Medicaid (54\% positive; $P=0.1$ ). However, when looking at route of administration, we noted a significant difference in the IVIg population in terms of cost perception based on payer. Significantly more IVIg 


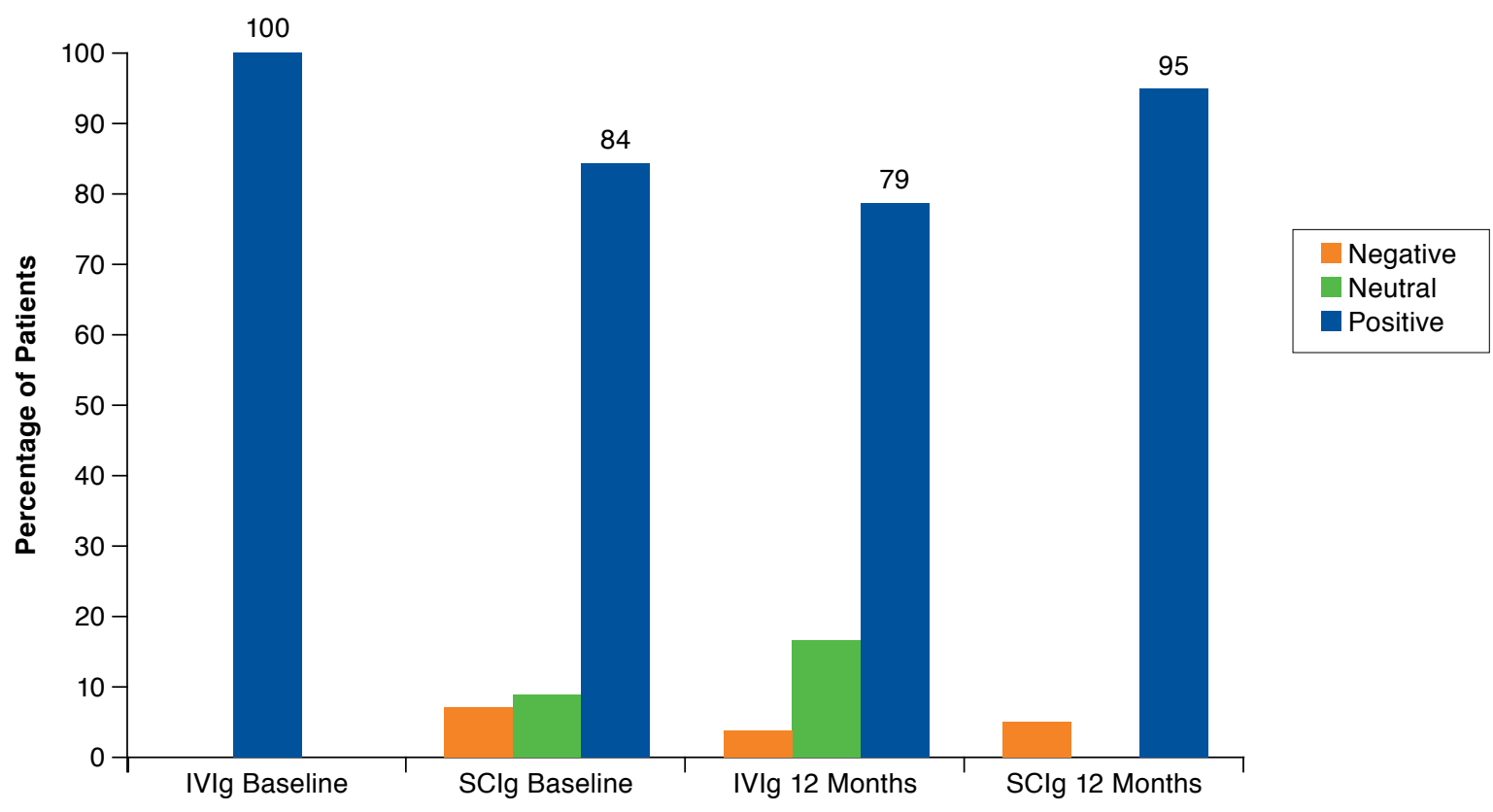

Ig=immunoglobulin; IVIg = intravenous immunoglobulin; SCIg= subcutaneous immunoglobulin.

patients who had a negative perception had private insurance (83\%, $P=0.03$ ), compared with the SCIg group, where those with a negative perception were evenly split between government (51\%) and private (49\%) payers (Figure 3).

\section{Overall Health Perception}

Using the SF-36 survey, we asked patients to rate their current overall health at baseline, 6 months, and 12 months. Patients scored their health on a scale of $1-5$, and we split their responses into "good health" $(1,2)$, "fair health" (3), and "poor health" $(4,5)$. By tracking individual responses at the different time points, we could determine within a population what the actual perceived change in health was across time. Baseline responses $(n=134)$ showed that $16 \%$ of patients reported good health; $44 \%$ reported fair health; and $40 \%$ reported poor health. At the 6-month time point, $92 \%$ of the patients in poor health at baseline showed a health improvement or no further decline (47\% improved, $45 \%$ no change). For those patients in good health at baseline, 52\% improved or stayed the same. Of those who did show a health decline, $75 \%$ still reported their health as fair. At the 12-month time point, we saw that for those who said their health was good at baseline, 31\% stayed at good; $38 \%$ went to fair; and 31\% went to poor. For those who said their health was fair at baseline, at the 12-month time point: $28 \%$ went to good; $53 \%$ stayed at fair; and 19\% went to poor. For those who reported poor health at baseline, at the 12-month time point: $9 \%$ said their health was now good; 33\% said fair; and 58\% remained at poor health (Figure 4).

\section{Discussion}

The IDEaL Patient Registry is focused on the long-term collection of clinical, quality-of-life, and health outcomes data for patients on Ig therapy. In our PID population of 367 patients, we had pronounced gender predominance towards women and patients in late adulthood. Our average age of start of treatment for Ig-naive patients was 9 years for pediatric patients, and 59 years for adult patients. These data suggest that unless diagnosed in mid-childhood, most of these patients did not get diagnosed until much later in life. Other reports have shown that in pediatric patients, the average delay in diagnosis is 5.8 years, ${ }^{5}$ and for adults diagnosed in a hospital setting, the average age was 35.3 years. ${ }^{7}$ Our average age was higher, which may reflect that most of our patients were diagnosed by community-based physicians, and their symptoms were milder and did not require hospitalization. While many may be asymptomatic, or minimally sick, some patients go through several decades of recurrent infections that can cause end-stage organ damage before starting on Ig therapy.

Patients can receive Ig therapy either through intravenous or subcutaneous administration. IVIg must be administered by a qualified health care provider, while patients can be trained to self-administer SCIg. Both routes are available 


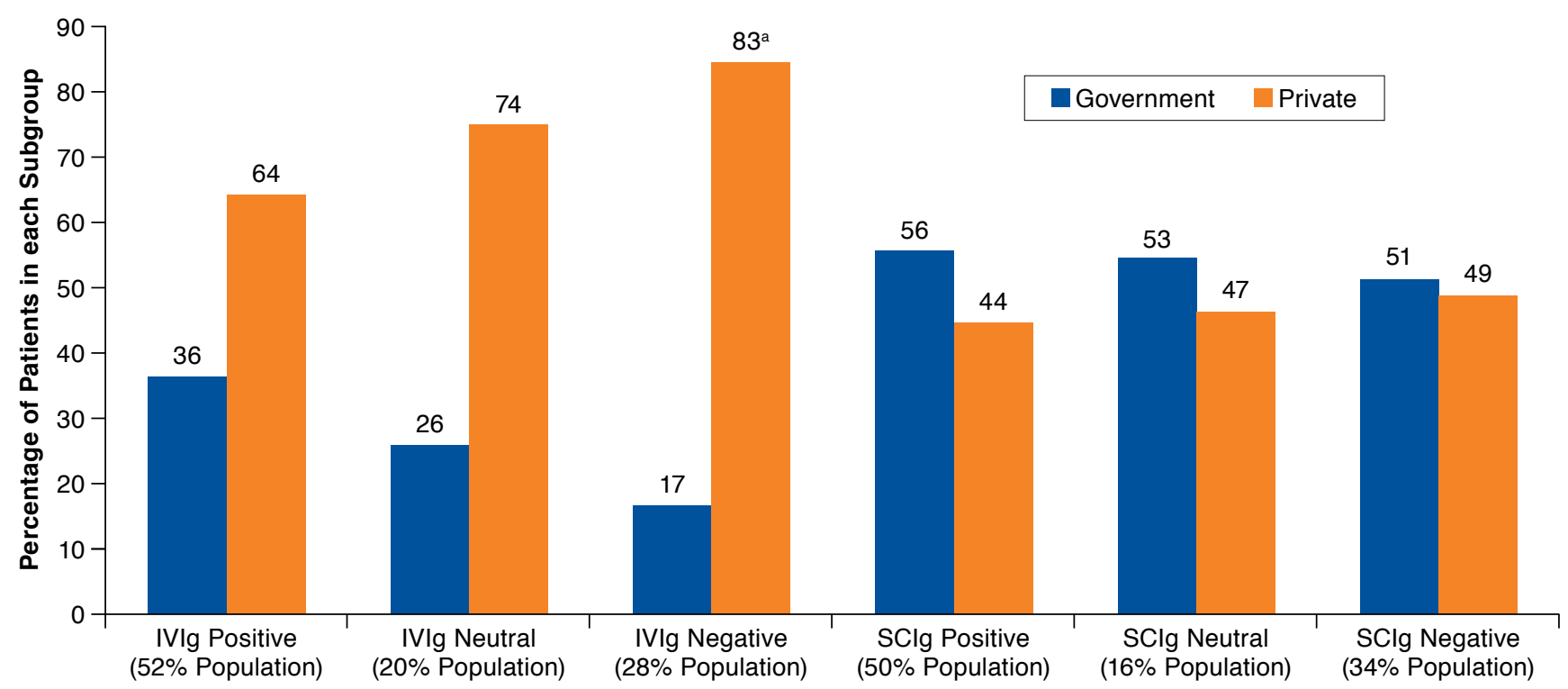

a Significantly more IVIg patients who had a negative perception had private insurance $(83 \%, \mathrm{P}=0.03)$ compared with the SCIg group where those with a negative perception were evenly split between government (51\%) and private (49\%) payers.

IVIg=intravenous immunoglobulin; SCIg=subcutaneous immunoglobulin; QOL=quality of life.

to patients at home. The majority of our patient population received SCIg (80\%) weekly, although 6\% of the SCIg patients were on an every-other-week schedule. Dosing for SCIg patients averaged about 10 grams per week, or $130 \mathrm{mg} / \mathrm{kg}$. This is on the lower end of the manufacturer-recommended dosing of $100-400 \mathrm{mg} / \mathrm{kg}$. ${ }^{8}$ For IVIg patients, dosing averaged 36 grams per month, or $472 \mathrm{mg} / \mathrm{kg}$. This was more in the middle of a manufacturer guideline for IVIg of $300-600 \mathrm{mg} / \mathrm{kg}$. ${ }^{9}$ These data agree with other publications that looked at home care use of Ig, in which more patients were noted on SCIg than IVIg and that there was an age-related dose function, where older patients were being given a lower dose in terms of overall $\mathrm{mg} / \mathrm{kg} .{ }^{10}$ Previous reports have also shown that higher Ig doses, and maintaining higher Ig levels through higher dosing, can lead to a decrease in infections. ${ }^{11,12}$

We did not note a large number of dose adjustments across the entire population. Only 30\% of SCIg patients and 34\% of IVIg patients had dose or frequency changes that altered their effective weekly or monthly dosing. For SCIg patients, the majority (60\%) of the dose adjustments were increases, while in IVIg patients, $42 \%$ of the adjustments were increases. Given the low number of dose changes in both routes, an opportunity may exist for pharmacy, nursing, and care providers to assess what steps are being taken to monitor patient dosages and what guidelines or thresholds are in place to ensure therapeutically effective dosing.
Patient-reported compliance rates were generally very high in this population. We noted a higher percentage of missed doses in the SCIg group compared with the IVIg group but a higher percentage of delayed doses in IVIg compared with SCIg. Given that a nurse visit is required to administer IVIg, it makes sense that there would be fewer missed doses, since a nurse would make sure to reschedule a dose, even if it was delayed. Since this is patient-reported compliance, it certainly would be of benefit to see how this compares with drug shipment data or other drug compliance methodologies. Annual Ig-level checks may represent the best option to not only help determine therapeutically relevant doses, but to detect noncompliance-affecting clinical outcomes and allow care providers to address the issue.

Patient perception of life quality and health outcomes is a critical measure for continued use and support of Ig therapy. In general, patients receiving Ig at home had a positive perception of the convenience of the treatment, with $86 \%$ having a positive response. This positive perception remained at the 12-month mark, with $92 \%$ of patients having a positive response. High marks for convenience are an important factor in therapy compliance and desire to continue on treatment. While we did not see any difference in the perception of convenience between IVIg and SCIg at either time point, we did note that IVIg showed a decrease in positive perception at 12 months compared with baseline, and SCIg showed an 

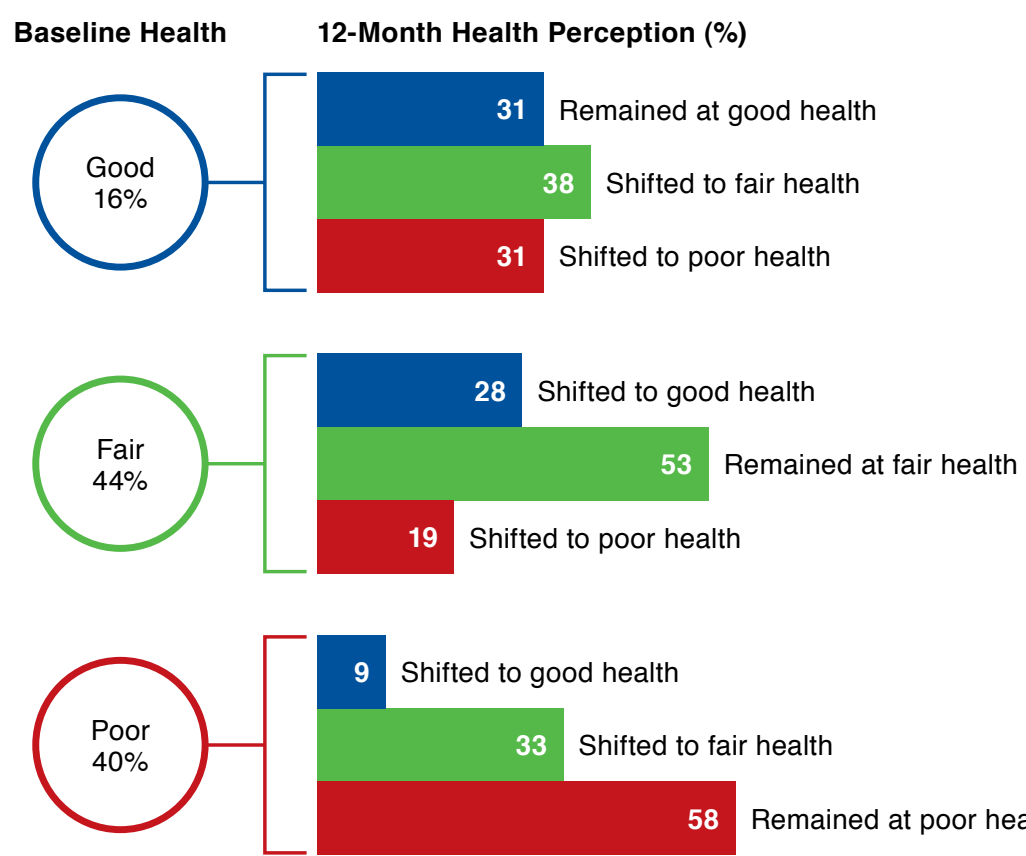

9 Shifted to good health

33 Shifted to fair health

58 Remained at poor health

aUsing an SF-36 survey, patients rated their current overall health at baseline, 6 months, and 12 months. Patients scored their health on a scale of 1-5; responses were split into "good health" (1, 2), "fair health" (3), and "poor health" (4, 5).

increase in perception of convenience at 12 months compared with baseline. Further analysis of this trend with a larger population and over a longer time period may show a more significant difference and may expose a significant difference between the routes. Overall, perception of convenience of either administration route at home remained high.

Ig therapy is an expensive specialty pharmacy product, and out-of-pocket costs can have a significant effect for those patients remaining on therapy long term. Overall cost perception was the most negative of the elements that were examined on the LQIQ survey, averaging a 4.3 out of 7 at baseline for all respondents, and a 4.5 out of 7 at 12 months. Across all time points, about half of the patients had a positive perception of the cost, while the other half was split between a neutral $(18 \%)$ or negative perception $(32 \%)$. We did not find a significant difference between administration routes in terms of cost perception nor did we find a difference in cost perception between those with private insurance or those on a government-sponsored health plan. A significant difference that we did note was that IVIg patients who had a negative perception were much more likely to have a private insurance plan, while SCIg patients who had a negative perception were evenly split. This discrepancy is likely because most government IVIg patients on service had a secondary or supplemental insurance plan that helped cover the out-of-pocket costs of treatment not covered by their primary government plan.

Overall perception of health while on treatment showed that how patients felt when they started treatment had an effect on how their health changed over time on treatment. The majority of those who reported their health as good at the start either stayed at good or declined only slightly to fair, while the majority of those in poor health at baseline stayed at poor. Given the common delays in diagnosing and starting treatment for PID patients, and that therapy can mitigate continued organ damage but not repair existing damage, the need for earlier intervention becomes critical to minimize associated comorbidities and maximize therapy outcomes.

\section{Limitations}

The IDEaL Patient Registry is an observational registry where patients must consent to participate. Our population, therefore, reflects the percentage of eligible patients who returned their consent form. Age, gender, diagnosis, and route of therapy may all show skewed results as a result of who returned their consent compared with the total population of eligible patients.

Compliance data are patient reported based on the pharmacist asking the patient if he or she had missed, delayed, or had any incomplete doses. Especially in the SCIg population where 
there is no other party involved in administration, patient recall of compliance, and willingness to admit noncompliance, may affect the reported rate.

Patient perception of health can be greatly affected by comorbidities. Since none of the survey questions were specific to symptoms of PID, it is possible that reported health declines or improvements could be related to another disease state.

\section{Conclusions}

Data from the IDEaL Patient Registry show that PID patients treated at home report good therapy compliance and good perception of treatment convenience and overall benefit to their health from their Ig treatments. Health improvement is affected by patient health status at the time treatment starts-patients who are sicker when they started Ig tend to report less improvement and greater declines than those who reported better health when they first started treatment. Our data showed that adult patients were aged almost 60 years on average when they first started treatment. While the presentation of their disease may have been milder, it still was preceded by multiple infections that affected patient health and organ damage. On average, patients also reported a slightly negative perception of the cost of their treatment. The insurance provider, either government or private, did not have a significant effect on patient perception of cost, except in the case of IVIg patients, where negative perception was significantly more common in patients with private insurance. The IDEaL Registry continues to collect long-term data on patients treated with Ig at home. Long-term data on diagnosing and outcomes in a real-world population represent a powerful tool to provide information back to health care providers to encourage a decreased time to diagnosis for PID patients, optimization of therapy, and improved patient outcomes.

\section{Authors}

SEAN KEARNS, PhD; LORETTA KRISTOFEK, BSN; WILLIAM BOLGAR, PharmD; and SAMANTHA KILE, BS, Coram/CVS specialty infusion services, Denver, Colorado. LUQMAN SEIDU, MD, Omni Allergy and Asthma, Atlanta, Georgia.

AUTHOR CORRESPONDENCE: William Bolgar, PharmD, Coram/CVS specialty infusion services, 555 17th St., \#1500, Denver, CO 80202.Tel.: 303.672.8745; E-mail:Registry@coramhc.com.

\section{DISCLOSURES}

No outside funding supported this study. Seidu was compensated by Coram Clinical Trials for acting as primary investigator and reviewing data.

Study concept and design were contributed by all the authors. Kearns, Kristofek, and Kiles collected the data, and data interpretation was performed by Kearns, Seidu, and Kristofek, along with Bolgar. The manuscript was written and revised primarily by Kearns, along with Kristofek, Bolgar, and Seidu.

\section{REFERENCES}

1. Marketing Research Bureau. US IVIG-SCIG forecast 2012-2017. Available at: http://marketingresearchbureau.com/list-of-reports/ivig-scig-forecast/. Accessed February 28, 2017.

2. Yong PL, Boyle J, Ballow M, et al. Use of intravenous immunoglobulin and adjunctive therapies in the treatment of primary immunodeficiencies: a working group report of and study by the Primary Immunodeficiency Committee of the American Academy of Allergy Asthma and Immunology. Clin Immunol. 2010;135(2):255-63.

3. Boyle JM, Buckley RH. Population prevalence of diagnosed primary immunodeficiency diseases in the United States. J Clin Immunol. 2007;27(5):497-502

4. Joshi AY, Iyer VN, Hagan JB, St Sauver JL, Boyce TG. Incidence and temporal trends of primary immunodeficiency: a population-based cohort study. Mayo Clin Proc. 2009;84(1):16-22.

5. Urschel S, Kayikci L, Wintergerst U, Notheis G, Jansson A, Belohradsky BH. Common variable immunodeficiency disorders in children: delayed diagnosis despite typical clinical presentation. J Pediatr. 2009;154(6):888-94.

6. Cunningham-Rundles $C$, Bodian $C$. Common variable immunodeficiency: clinical and immunological features of 248 patients. Clin Immunol. 1999;92(1):34-48.

7. Chapel H, Lucas M, Lee M, et al. Common variable immunodeficiency disorders: division into distinct clinical phenotypes. Blood. 2008;112(2):277-86.

8. HIZENTRA, immune globulin subcutaneous (human), 20\% liquid. CSL Behring AG. Revised October 2016. Available at: http://labeling.cslbehring. com/PI/US/Hizentra/EN/Hizentra-Prescribing-Information.pdf. Accessed February 28, 2017.

9. GAMMAGARD LIQUID, immune globulin infusion (human) 10\%. Baxter International. April 2014. Available at: http://www.baxter.com/products/biopharmaceuticals/downloads/gamliquid_PI.pdf.

10. Huang F, Feuille E, Cunningham-Rundles C. Home care use of intravenous and subcutaneous immunoglobulin for primary immunodeficiency in the United States. J Clin Immunol. 2013;33(1):49-54

11. Haddad E, Berger M, Wang EC, Jones CA, Bexon M, Baggish JS. Higher doses of subcutaneous $\operatorname{Ig} G$ reduce resource utilization in patients with primary immunodeficiency. J Clin Immunol. 2012;32(2):281-89.

12. Orange JS, Grossman WJ, Navickis RJ, Wilkes MM. Impact of trough $\operatorname{IgG}$ on pneumonia incidence in primary immunodeficiency: a meta-analysis of clinical studies. Clin Immunol. 2010;137(1):21-30. 